\title{
Fetus Electrocardiogram by using Neuro-Fuzzy Systems and Remote Sensing through OFDM
}

\author{
Pradeep Kumar, A.Shiva Prasad
}

\begin{abstract}
The fetus electrocardiogram (FECG) can be obtained after 21 days of pregnancy. It will be also useful as noninvasive technique to check whether women are pregnant or not. So early diagnosis can be done for any cardiac defects. The FECG segment from mother stomach recording and reference thoracic maternal electrocardiogram (MECG) signal is acquired by counterfeit neural system fluffy surmising framework (ANFIS). The wavelet change is utilized for upgrade. The symmetrical recurrence division multiplexing (OFDM) is helpful for transmission in remote detecting.
\end{abstract}

Key words: OFDM, Neural Network, MECG, FECG, Fuzzy logic, SNR, Wavelet transform \& Membership function.

\section{INTRODUCTION}

Fetus ECG is a unique method available to see the electrocardiography of fetus. Regularly two frameworks are being utilized: the main technique is immediate, in which cathode is appended with scalp of the foetus and the other is indirect, in this signal is sensed from the wall of the maternal stomach. The former technology is more risky and can only be done during the labour.

Electrocardiogram(ECG) give a realistic chronicle of the electrical powers created by the heart. In this paper, we propose non-intrusive procedure, FECG is obtained from contaminated abdomen signal through ANFIS. In order to achieve this for remote area, OFDM is used. The Paper has been organized as: Dialog of provision II is about the proposed framework, area III talk about outcomes and segment IV comprises of end.

\section{THE PROPOSED SYSTEM}

In our proposition framework, we will have two sign. That prompts two pieces of information. One sign is gotten from thoracic and another is acquired from the stomach zone of the pregnant woman. Non-linear transform is used on MECG.

The stomach sign is comprise with three sign: One is a distorted form as it ventures out from the chest to the guts, another is the fetal ECG and a third is because of impacts of movement artefatcs. The stomach sign can be communicated as the entirety of a distorted adaptation of the maternal ECG and some commotion with the fetal ECG.

The twisting of the maternal ECG segment occurs because of some nonlinear transformation[7], [8] as it goes to the stomach region from chest. The thoracic sign is overwhelmingly maternal, and thus the fetal part in it is disregarded.

Revised Manuscript Received on September 10, 2019.

Dr. Pradeep Kumar, Associate Professor, Dept. of ECE, CMRIT Hyderabad, Telangana, India.

A. Shiva Prasad, Assistant Professor, Dept. of ECE, CMRIT Hyderabad, Telangana, India.
In our strategy, we do as such by an ANFIS connect with numerous information and one yield. The sources of info are here the MECG signal and a limited number of its subsidiaries or deferrals. The ANFIS system will locate a nonlinear change. The privilege ANFIS system will be useful to get a gauge of the maternal part. An underlying arrangement of participation capacities is utilized for preparing. The ANFIS yield is subtracted from the tainted sign to acquire the baby signal. The FECG is upgraded by utilizing wavelet change [5] to get the more clear FECG.

The OFDM technique is used for transmission. The patient in remote area also can be treated in well manner. The work is carried for synthetic data by using Matlab. The signal to noise ratio(SNR) is maintained high in compare to previous methods.

\section{RESULTS}

The abdominal ECG (AECG) from mother abdomen is shown in fig.1. It has fetus component as well as mother thoracic component. It is desire to obtain fetus component separately for diagnosis.

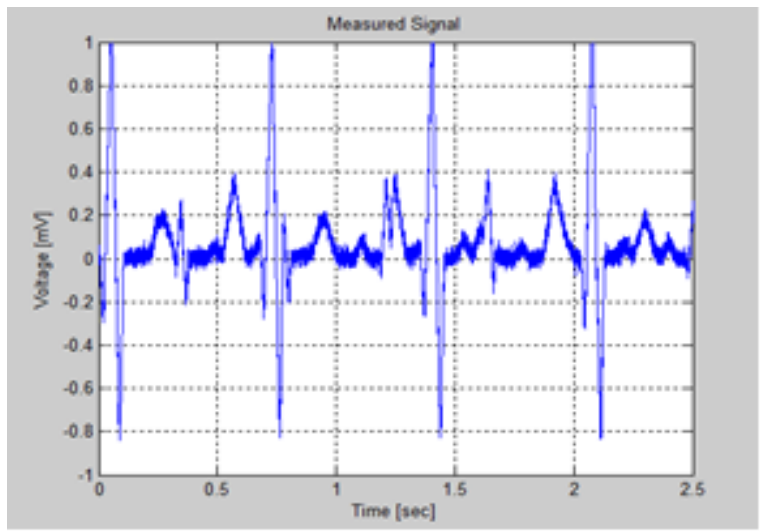

Fig.1: AECG obtained from mother abdomen 


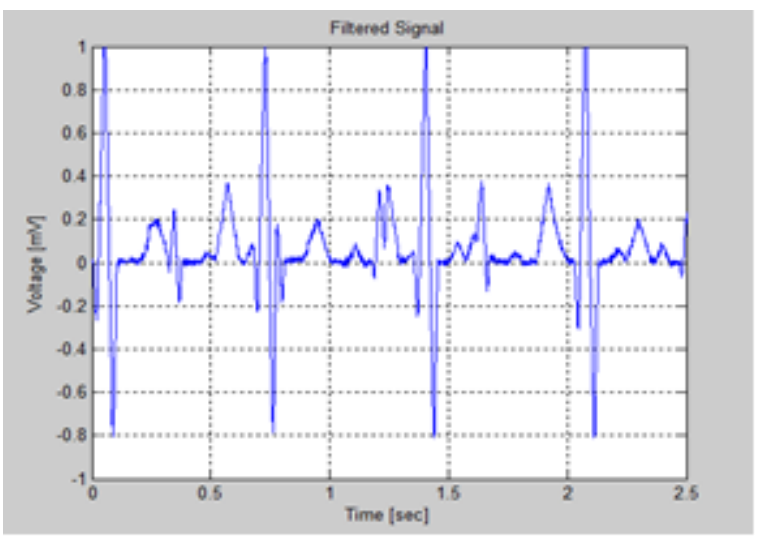

Fig.2: Filtered Signal of AECG

Before that signal is filtered to remove motion artefacts. Wavelets are used for pre and post de-noising.

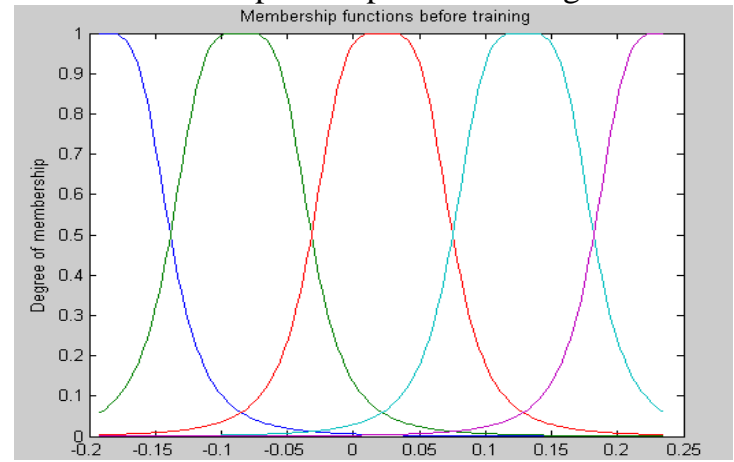

Fig.3: Membership function

The gbell membership function is applied before training and after too, which is shown in fig.3. In the transmission QPSK (Quadrature Phase Shift Keying) is adopted due to limitations of other techniques. The performance graph $\mathrm{b} / \mathrm{w}$ SNR \& BER(Bit Error Rate) is shown in fig.4.

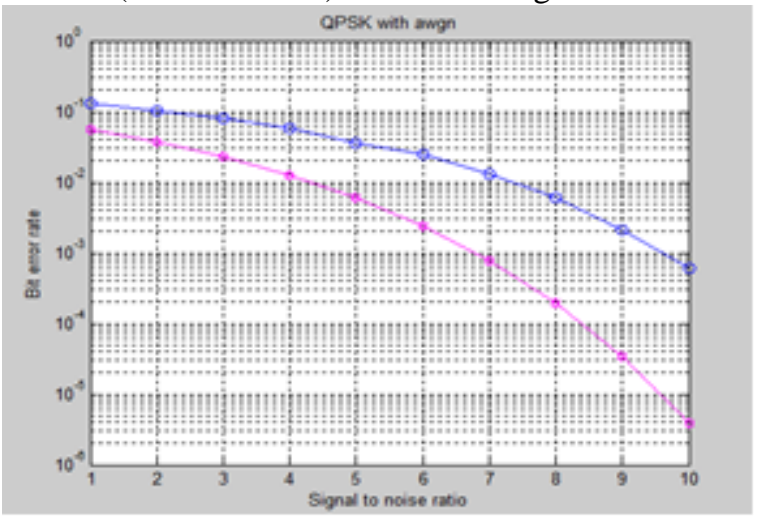

Fig.4: SNR vs BER

The mother wavelets of db6 is selected for better performance on basis of repeated results, which is given in fig.5. OFDM carriers are shown in fig.6. The phase of modulated signal is shown in fig.7. We are able to distinguish even during QRS portion of the ECG.

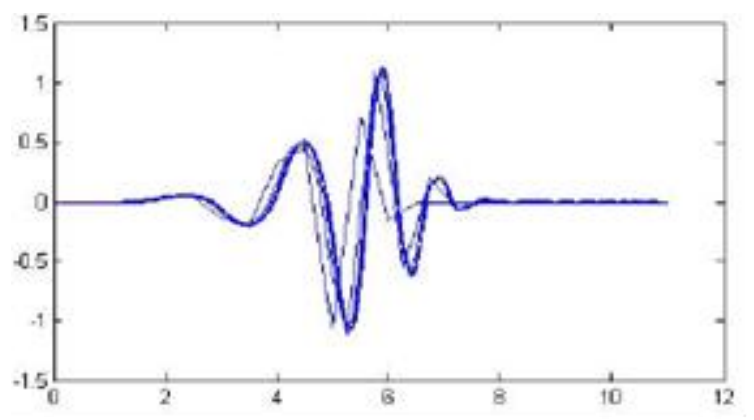

Fig.5: db6 Mother Wavelet Function

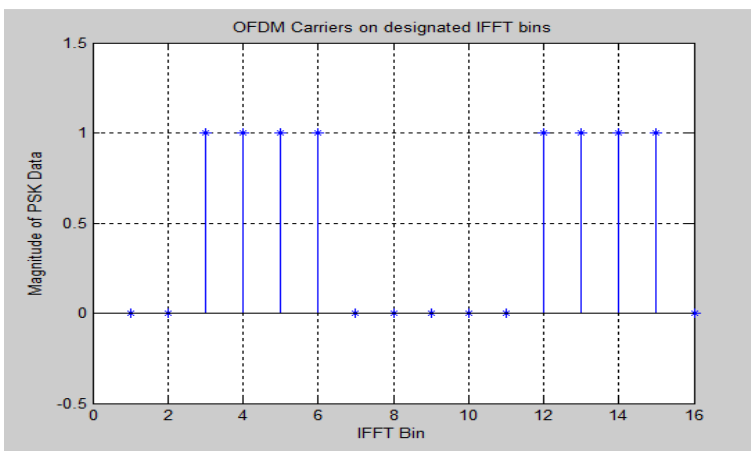

Fig.6: OFDM Carriers on IFFT bins

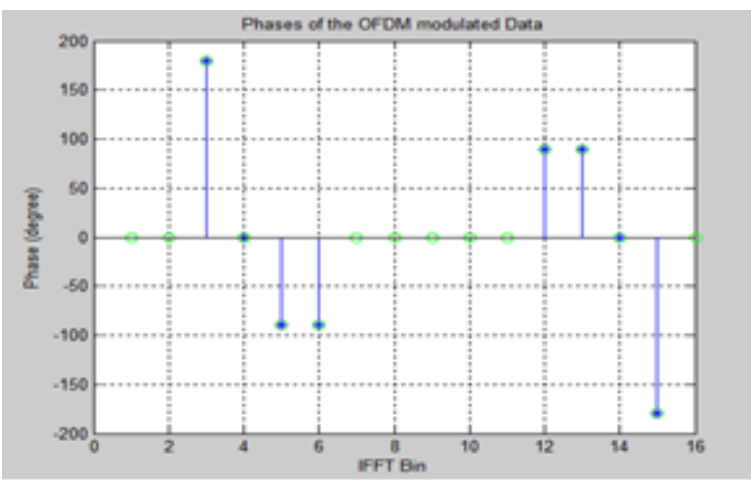

Fig.7: Phase of modulated data

The measured signal is de-noised and residual of FECG is obtained, which is shown in fig. 8 and fig. 9 respectively.

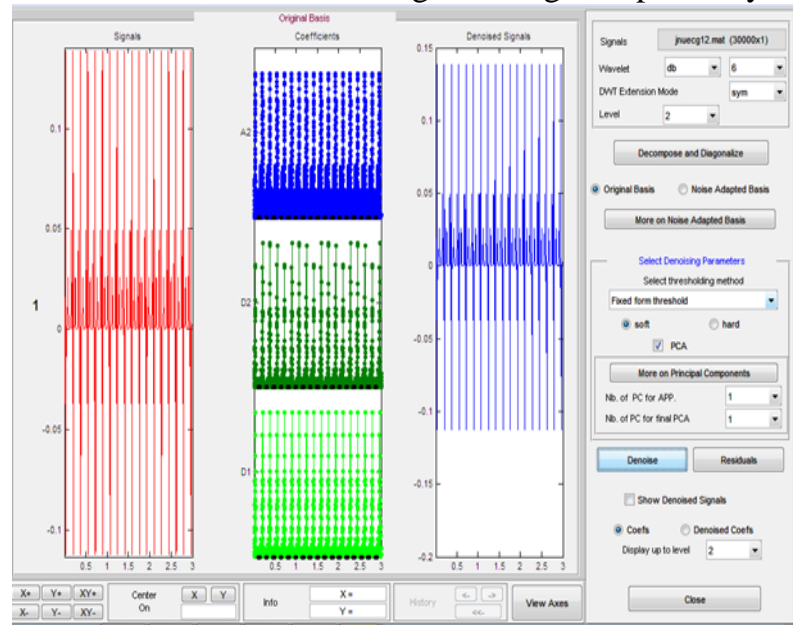

Fig.8: De-noising of measured AECG 


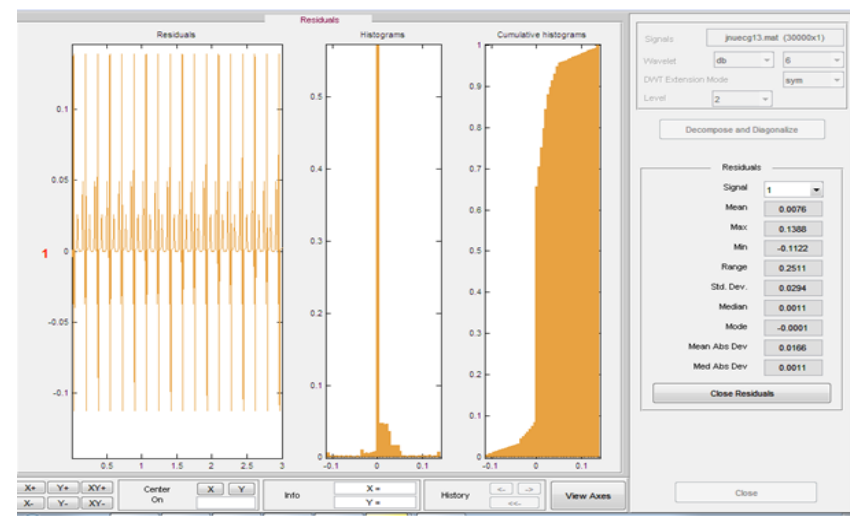

Fig.9: Residuals of FECG

Finally fetus ECG is obtained from contaminated signal. Which is shown in fig.10. On the basis of this we can diagnosis condition of fetus inside abdomen before birth with this non-invasive technique.

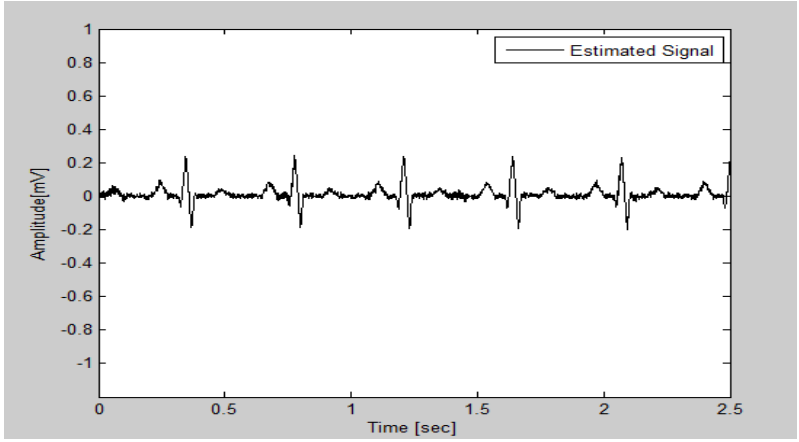

Fig.10: The obtained Fetus ECG from contaminated signal by gbellmf

\section{CONCLUSION}

The acquired outcome is relatively superior to past different strategies particularly in the district of QRS. Various kinds of enrollment capacities and wavelets change's diff. levels are attempted, in which best one is chosen to get want result.

\section{ACKNOWLEDGEMENT}

We are thankful to Dr. Chanda Jha and Mrs. Anjana Walia for their direction and supports all through the work.

\section{REFERENCES}

1. Giulia Da Poian ; Riccardo Bernardini ; Roberto Rinaldo, Separation and Analysis of Fetal-ECG Signals From Compressed Sensed Abdominal ECG Recordings, IEEE Transactions on Biomedical Engineering, Year: 2016, Volume: 63, Issue: 6, Pages: 1269 - 1279, IEEE Journals \& Magazines.

2. Udit Satija ; Barathram Ramkumar ; M. Sabarimalai Manikandan, Automated ECG Noise Detection and Classification System for Unsupervised Healthcare Monitoring, IEEE Journal of Biomedical and Health Informatics, Year: 2018, Volume: 22, Issue: 3,Pages: 722 - 732,IEEE Journals \& Magazines.

3. Chunyu Tan ; Liming Zhang ; Hau-tieng Wu, A Novel Blaschke Unwinding Adaptive Fourier Decomposition based Signal Compression Algorithm with Application on ECG Signals, IEEE Journal of Biomedical and Health Informatics, Year: 2018, ( Early Access ),Pages: 1 1,IEEE Early Access Articles.

4. Anoop, S.S.V.K.K. ; Prabhu, Ch.M. ; Penumala, S.K. Detection of QRS Complex of Fetal ECG Using
Biologically Inspired Soft-Computing Tools, India Conference (INDICON), 2009 Annual IEEE

5. Hasan, M.A. ; Reaz, M.B.I. ; Ibrahimy, M.I. Fetal electrocardiogram extraction and R-peakdetection for fetal heart rate monitoring using artificial neural network and Correlation, Neural Networks (IJCNN), The 2011 International Joint Conference on Digital Object Identifier: 10.1109/IJCNN.2011.6033193 Publication Year: 2011 , Page(s): $15-20$

6. Chengyu Liu ; Peng Li, Systematic methods for fetal electrocardiographic analysis: Determining the fetal heart rate, RR interval and QT interval Computing in Cardiology Conference (CinC), 2013 , Publication Year: 2013 , Page(s): $309-312$

7. Christov, I. ; Simova, I. ; Abacherli, R. Cancellation of the maternal and extraction of the fetal ECG in noninvasive recordings, Computing in Cardiology Conference (CinC), 2013 Publication Year: 2013, Page(s): 153 - 156.

8. Xu-Wilson, M. ; Carlson, E. ; Limei Cheng ; Vairavan, S. Spatial filtering and adaptive rule based fetal heart rate extraction from abdominal fetal ECG recordings Computing in Cardiology Conference (CinC), 2013, Publication Year: 2013 , Page(s): 197 - 200

9. Guojun Li ; Xiaoping Zeng ; Xiaona Zhou ; Qilie Liu Robust adaptive fetal heart rate estimation for singlechannel abdominal ECG recording, Biomedical Engineering and Informatics (BMEI), 2012 5th International Conference on Digital Object Identifier, Publication Year: 2012 , Page(s): 587 - 591

10. Llamedo, M. ; Martin-Yebra, A. ; Laguna, P. ; Martinez, J.P., Noninvasive fetal ECG estimation based on linear transformations Computing in Cardiology Conference (CinC), 2013 ,Publication Year: 2013 , Page(s): 285 288 